\title{
NeuroImage
}

\section{Segmentation of brain structures in presence of a space-occupying lesion}

\author{
Claudio Pollo, ${ }^{\mathrm{a}, \mathrm{b}, *}$ Meritxell Bach Cuadra, ${ }^{\mathrm{b}}$ Olivier Cuisenaire, ${ }^{\mathrm{b}}$ \\ Jean-Guy Villemure, ${ }^{\mathrm{a}}$ and Jean-Philippe Thiran ${ }^{\mathrm{b}}$ \\ ${ }^{a}$ Department of Neurosurgery, Centre Hospitalier Universitaire Vaudois (CHUV), Lausanne, Switzerland \\ bignal Processing Institute, Ecole Polytechnique Fédérale Lausanne (EPFL), Lausanne, Switzerland
}

Received 8 April 2004; revised 13 September 2004; accepted 6 October 2004

Available online 26 November 2004

Brain deformations induced by space-occupying lesions may result in unpredictable position and shape of functionally important brain structures. The aim of this study is to propose a method for segmentation of brain structures by deformation of a segmented brain atlas in presence of a space-occupying lesion. Our approach is based on an a priori model of lesion growth (MLG) that assumes radial expansion from a seeding point and involves three steps: first, an affine registration bringing the atlas and the patient into global correspondence; then, the seeding of a synthetic tumor into the brain atlas providing a template for the lesion; finally, the deformation of the seeded atlas, combining a method derived from optical flow principles and a model of lesion growth. The method was applied on two meningiomas inducing a pure displacement of the underlying brain structures, and segmentation accuracy of ventricles and basal ganglia was assessed. Results show that the segmented structures were consistent with the patient's anatomy and that the deformation accuracy of surrounding brain structures was highly dependent on the accurate placement of the tumor seeding point. Further improvements of the method will optimize the segmentation accuracy. Visualization of brain structures provides useful information for therapeutic consideration of space-occupying lesions, including surgical, radiosurgical, and radiotherapeutic planning, in order to increase treatment efficiency and prevent neurological damage.

(C) 2004 Elsevier Inc. All rights reserved.

Keywords: Space-occupying lesions; Neurological damage; Atlas-based MR segmentation algorithms

\section{Introduction}

Precise segmentation of functionally important brain anatomical structures and/or areas is of major interest in the minimal invasive approaches to brain space-occupying lesions, including tumors and

* Corresponding author. Department of Neurosurgery, CHUV, Rue du Bugnon, CH-1011 Lausanne, Switzerland. Fax: +41 213142605.

E-mail address: claudio.pollo@chuv.hospvd.ch (C. Pollo)

Available online on ScienceDirect (www.sciencedirect.com). vascular malformations. It aims to reduce the morbidity or mortality and to improve the outcome of surgical, radiosurgical, or radiotherapeutic management of such lesions. Multimodal investigations such as functional MRI, PET, and SPECT scans have certainly contributed to a more accurate localization of structures of interest but may be limited by their low anatomical resolution. Despite the spatial information and higher anatomical resolution provided by MRI and three-dimensional (3D) reconstructions performed by image-guided systems, precise visual segmentation may be inaccurate when anatomic structures are shifted and deformed.

Atlas-based MR segmentation algorithms have already shown to be reliable when performed on nonshifted brain structures (Bach Cuadra et al., 2001; Dawant et al., 1999a) but are still of limited use when a space-occupying lesion induces brain deformations that are yet not clearly predictable.

We report our preliminary experience of brain structures segmentation in the presence of a space-occupying lesion based on a brain atlas deformation. The mathematical and biophysical concepts of our deformable model are explained. Its advantages and limits are discussed in the field of future applications in neurosurgery, radiotherapy, and radiosurgery.

\section{Materials and methods}

Dataset

The MR datasets of two patients with meningiomas were selected for this study. In each case, a 3D T1-weighted magnetization-prepared rapid acquisition gradient echo (MPRAGE) MR sequence with gadolinium was performed (Magneton $1.5 \mathrm{~T}$, Siemens, Erlangen, Germany) with the following parameters: 128 sagittal slices of $1.25 \mathrm{~mm}$. Field of view $280 * 280 \mathrm{~mm}$; matrix size $256 * 256$; voxel size $1.09375 \times 1.09375 \times 1.25 \mathrm{~mm}$.

The digitized brain atlas used in this study was kindly provided by the Surgical Planning Laboratory (SPL) of the Harvard Medical School (Kikinis et al., 1996). 


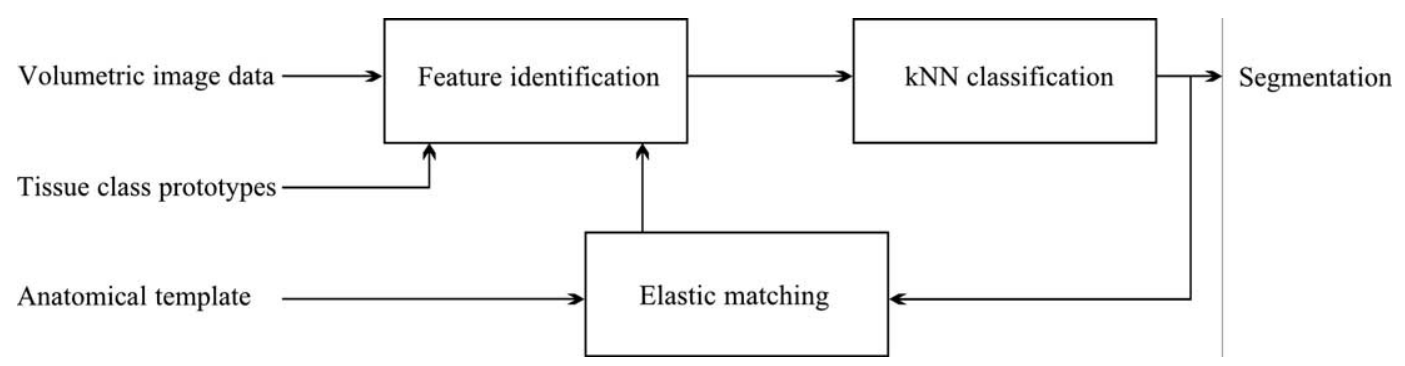

Fig. 1. Scheme of the ATM SVC algorithm.

\section{Data processing}

An affine transformation was applied to the atlas in order to reach a global matching with the patient's brain without consideration of the brain deformation induced by the lesion. The tumor was then automatically segmented from the patient MRI. A seeding point representing the supposed seed of the tumor was then identified and placed on the atlas, and a nonrigid deformation algorithm using our model of tumor growth was applied until reaching the previously segmented limits of the tumor, with an a priori radial growth assumption. A registration algorithm based on the demons method was applied outside the limits of the tumor for the brain deformation.

\section{Affine transformation}

As proposed by Cuisenaire et al. (1996), the global transformation from the patient image to the atlas is modeled as a threedimensional affine transform, that is, $y=A x+b$ with $A$ as a $3 \times 3$ matrix and $b$ as a $3 \mathrm{D}$ vector. The 12 parameters of this transform are optimized in order to minimize the distance between the patient and atlas cortical surfaces, both segmented using mathematical morphology operators. This distance between surfaces is defined as the mean square value, over all points of moving surface, of the distance to the nearest point from the reference surface. These are efficiently precomputed using a fast Euclidean distance transform algorithm.

\section{Lesion segmentation}

The patient's lesion needs to be segmented in order to specify the volume in which the model of tumor growth will be applied. To this purpose, we use a variant of the Adaptive Template Moderated Spatially Varying Statistical Classification (ATM SVC) algorithm proposed by Warfield et al. (2000). This algorithm uses both image and anatomical information, embedding them in a high dimensionality space in which a k-Nearest Neighbors (k-NN) classification is performed. One dimension of this feature space is the image intensity. The other dimensions are the distances to the structures from a brain atlas warped onto the corresponding structures classified from the patient image. The algorithm is implemented in a hierarchical way, so that the dimensionality of the feature space and the number of classified structures increase progressively at each level:

- Level 1: The intensity feature is used for the classification into the brain and the background classes.

- Level 2: The intensity and distance to the brain surface are used to classify the ventricles, brain, and background.

- Level 3: Intensity, distance to the brain, and distance to the ventricles are used for the classification into lesion, ventricles, brain, and background.

- Level 4: Intensity, distance to de brain, distance to the ventricles, and distance to the lesion contour are used for the final classification into lesion, ventricles, brain, and background.

In the first level, the classification is done by simply binarization of the image. In the levels 2, 3, and 4, the process represented in Fig. 1 is applied. We use the demons algorithm (Thirion, 1998) as elastic matching instead of the one used in studies of Kaus et al. (1999) and Warfield et al. (2000). Note that the distance to the brain surface and to the ventricles is computed from the registered atlas, while the distance to the lesion is computed from the classified patient (see the Level 3 in Fig. 2).

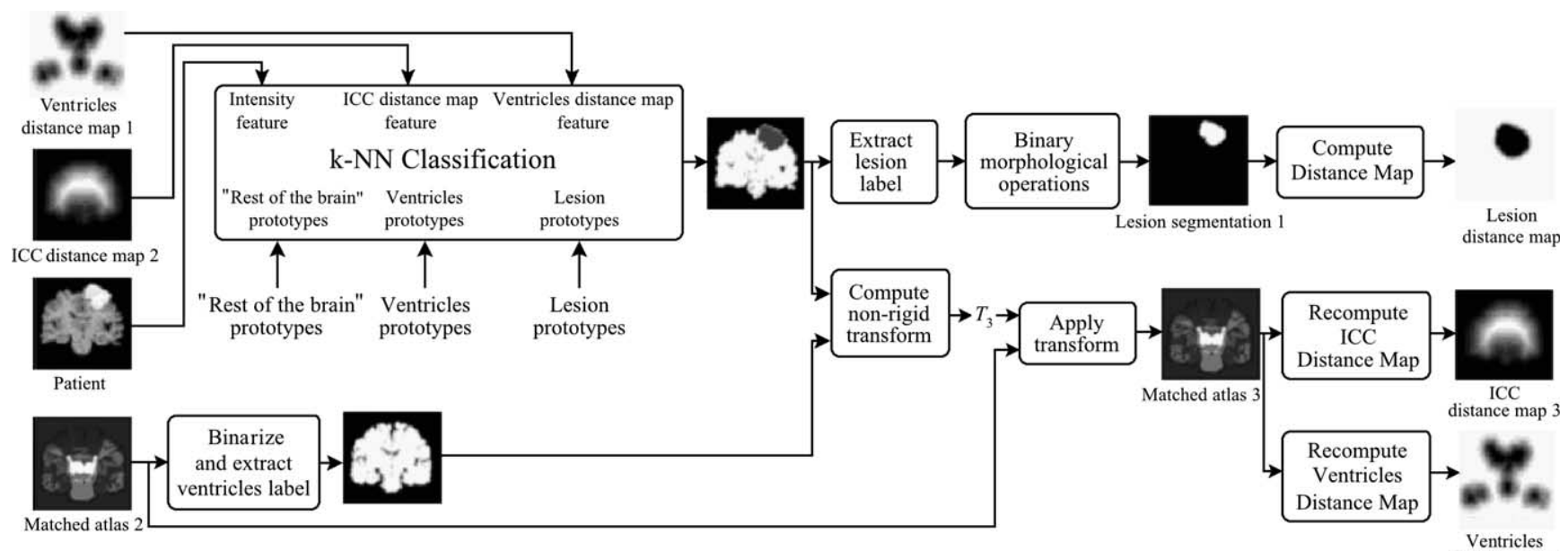


Moreover, the k-NN classifier is trained by manually choosing on MR images 100 prototypes per class (lesion, ventricle, and brain classes).

\section{Atlas seeding}

After the affine transformation, the atlas and patient volumes are globally in correspondence except in regions that have been drastically deformed by the tumor. We proceed to the atlas seeding by manually selecting the point of origin of the tumor growth in the affine-registered brain atlas.

Both our previous work (Bach Cuadra et al., 2002) and that of other groups, namely, Dawant et al. (1999b) group, use an extended seed in order to drive the tumor deformation. It makes the positioning of the seed a relatively easy task but unfortunately masks atlas structures under the seed. In this paper, the singlevoxel seed induces no masking, but-as will be discussed-the selection of the correct seed location requires anatomical and biological knowledge of tumor growth.

\section{Nonrigid deformation using a model of lesion growth (MLG algorithm)}

At this point, the affine registration ensures that the small displacement assumption is respected in the region of the brain that is far from the tumor. Meanwhile, the segmentation of the tumor volume and the manual selection of the tumor seed provide an adequate model for the tumor and its influence on immediately surrounding tissues. The nonrigid deformation method we propose distinguishes between those two areas fixed from the lesion segmentation: outside the lesion, where the demons algorithm is applied, and inside the lesion, where a simple model of lesion growth is applied.

Demons algorithm. This algorithm approaches the problem of image matching as a diffusion process, in which object boundaries in the reference image $F$ are viewed as semipermeable membranes. The other (so-called floating) image $G$ is considered as a deformable grid and diffuses through these interfaces driven by the action of effectors situated within the membranes. These effectors are also called demons by analogy with Maxwell's demons.

In this paper, the instantaneous force (velocity) for each demon point $\vec{p}$, at the iteration $i+1$ is

$\vec{v}^{i+1}(\vec{p})=\frac{\left(G\left(\vec{p}+\vec{D}^{i}(\vec{p})\right)-F(\vec{p})\right)}{|\vec{\nabla} F(\vec{p})|^{2}+\left(G\left(\vec{p}+\vec{D}^{i}(\vec{p})\right)-F(\vec{p})\right)^{2}} \vec{\nabla} F(\vec{p})$,

where $F($.$) and G($.$) are the image intensities and \vec{D}^{i}$ is the current displacement field. Thus, there is a displacement in the direction of the reference image gradient, provided there is both a difference in image intensities and a reference image gradient different from zero.

The deformation field is then computed from the instantaneous velocity by assuming that the two images to match are two frames separated by a unit of time:

$\vec{d}^{i+1}=\vec{D}^{i}+\vec{v}^{i+1} \cdot \Delta t$,

where $\Delta t=1$.

Note that Eq. (1) is asymmetrical; that is, it gives different results depending on which image is chosen as the reference and which is chosen to be floating. In (Thirion, 1995), a solution to provide bijectivity to the demons algorithm is presented and therefore to provide a way of finding the inverse transformation. This is done by computing at each iteration both the direct deformation field $\vec{d}_{\text {direct }}$, from Eqs. (1) and (2) and the inverse deformation field, $\vec{d}_{\text {inverse }}$, also from Eqs. (1) and (2) but replacing $F$ instead of $G$ and vice versa. Then, a residual vector field $R=$ $\vec{d}_{\text {direct }}+\vec{d}_{\text {inverse }}$ is equally distributed onto the two deformation fields:

$\vec{D}_{\text {direct }}^{*_{i+1}}=\vec{d}_{\text {direct }}^{i+1}-\frac{\vec{R}}{2}$

$\vec{D}_{\text {inverse }}^{*_{i+1}}=\vec{d}_{\text {inverse }}^{i+1}-\frac{\vec{R}}{2}$

In this approach, global smoothness of the total displacement field is implicitly enforced. Locally similar displacements for nearby voxels are imposed by smoothing both direct and inverse displacement fields with a Gaussian filter, that is, $\overrightarrow{D_{\alpha}}=\vec{D}_{\alpha}{ }^{*} i+1 \circ G(\sigma)$, where $\vec{D}^{*}$ is the deformation field at the current iteration, $\alpha$ refers to direct and inverse, $G(\sigma)$ is the Gaussian filter with standard deviation $\sigma$, and $\vec{D}$ is the regularized deformation field that will be used in Eq. (1) the next iteration. The choice of the smoothing parameter $\sigma$ of the filter is a critical issue that has been studied in Thirion (1995). We apply the nonrigid registration as an elastic process, and therefore, we also call $\sigma$ the elasticity parameter.

Model of lesion growth algorithm. Inside the lesion, the tumor growth model assumes a radial growth of the tumor from the tumor seed, that is,

$\vec{v}_{\text {lesion }}^{i+1}(\vec{p})=\frac{\overrightarrow{D M}}{N_{i t}}$

$\underset{\text { where }}{\vec{D}} \vec{v}_{\text {lesion }}$ is the instantaneous velocity inside the lesion area, $\overrightarrow{D M}$ is the distance from the corresponding point $\vec{p}$ to the seed, and $N_{\text {it }}$ is the number of iterations of the deformation algorithm that have to be performed. Then, the deformation field $\vec{d}_{\text {lesion }}^{i+1}$ is computed similarly as in Eq. (1). The bijectivity inside the lesion area is ensured by forcing $\vec{v}_{\text {direct }}=-\vec{v}_{\text {inverse }}$.

This model allows the points inside the tumor to converge towards the seed voxel, while remaining simple and allowing any number of iterations to take place outside the tumor volume.

The displacement vector computed at every voxel using either the demons force (1) or the lesion growth model (4) is regularized by an adaptive Gaussian filter to avoid possible discontinuities. Three areas are arbitrarily considered: (a) inside the lesion area, (b) close to the lesion (within $10 \mathrm{~mm}$ of the tumor) where large deformations occur, and (c) the rest of the brain. In (a), regularization of the deformation vector field is not necessary because the vector field induced by (2) is highly regular and the continuity is ensured, explaining why $\sigma=0$ inside this area. In (b) (including the tumor contour), the largest deformations are found due to the tumor growth. Then, it is necessary to allow large elasticity; that is, $\sigma$ should have a small value, typically $0.5 \mathrm{~mm}$. In (c), deformations are smaller, due primarily to interpatient anatomical variability. A larger $\sigma$ proves to be more accurate $(0.8 \mathrm{~mm})$, as it simulates a more rigid transformation.

Notice that the algorithm is implemented in a multiscale way: The number of iterations is arbitrarily fixed to $256+128+32+16$ 
from low to high resolution scale. A first match is made with down-sampled images, and the resulting transformation is upsampled to initialize the next match with finer image resolution.

\section{Control of the deformation accuracy}

After registering the atlas and patient MRIs, the same deformation is applied to the labeled structures of the atlas including ventricles, thalamus, and striatum and the patient's MRI. Once deformed, the labeled structures were projected on the patient's MRI.

For each case, one coronal and one sagittal slices crossing the center of the tumor as well as one axial slice located in the vicinity of the tumor and showing the structures of interest were selected after each deformation and compared to the corresponding structures visible on the patient's MRI. The accuracy of brain deformation and projected deformed labeled structures of the atlas was assessed by two investigators (one neurosurgeon and one engineer).

To evaluate the influence of the seeding point position, we created a synthetic atlas from the patient's MRI by mirroring the patient's healthy hemisphere. This synthetic atlas is as similar as possible to the patient's MRI in terms of interpatient variability, so that the remaining deformation between this synthetic atlas and the patient should only compensate for the tumor growth. MLG registration between the synthetic patient-atlas and the patient was applied for six different initial positions of the seed voxel chosen.

\section{Results}

\section{Deformed atlas images and deformation field}

The MLG algorithm has been run using the parameters described previously. In Fig. 3(left), the one-voxel seeded atlas is shown. Fig. 3(middle) represents one deformed atlas image after reaching the limits of the tumor, and Fig. 3(right) shows the deformation field.

\section{Seeding point position}

Seed position is a critical point of the MLG since it simulates where the tumor has begun to grow, and different choices of position may lead to very different results. The resulting deformation for each seed position is shown in Fig. 4. In each simulation, the original patient and the resulting deformed synthetic atlas-patient are displayed. The seed position is represented by a little sphere.

The areas where the MLG method has resulted in accurate deformations have been marked using green arrows, while inaccurate deformed areas are shown with red arrows. From a biological point of view, the most probable tumor seed takes place in the middle of area representing the dural attachment of the tumor. Even if this seed position seems to result in accurate displacement of the underlying brain tissue, it has also led to increased deformation of the midsagittal region. When starting with other seed positions, more accurate deformations have been obtained for the parasagittal region, whereas underlying gyri have not correctly been displaced.

When comparing deformation results for structures located far from the tumor (ventricles, basal ganglia, thalamus), little variability has been detected related to the seed position (Fig. 5).

\section{Global segmentation results}

Fig. 6 shows the final coregistration with the patient's MRI with the following labeled structures of the deformed brain atlas projected to the patient's image: the tumor (in red), the ventricles (in green), the thalamus (in yellow), and the striatum (in dark blue). A global best accuracy was obtained in patient 1 when the seed was placed near the superior sagittal sinus. In patient 2 , the best seed position was found in the parasellar region.

\section{Discussion}

Several approaches can be proposed for the treatment of brain space-occupying lesions, including surgery, radiotherapy, and radiosurgery, alone or in combination. Successful treatment of brain lesions like tumors and vascular malformation is mainly determined by the extent of removal and preservation of functionally important brain structures. Therefore, neurosurgical, radiosurgical, as well as radiotherapeutical planning of brain lesions should be performed in respect with these two objectives. Visualization of important brain structures on medical images is essential to increase the extent of lesion removal and avoid neurological complications resulting from brain damage induced by either direct surgical aggression and/or radionecrosis. Thus, the reliability of automatic segmentation of brain structures is of major importance to provide useful information for treatment consid-

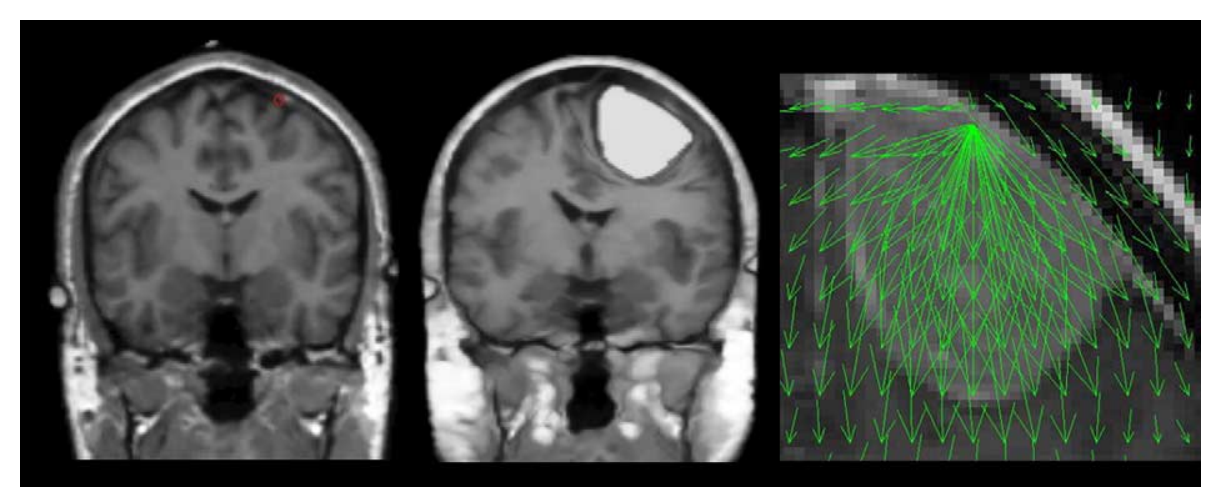

Fig. 3. (Left) Placement of the seed (red circle) on the atlas after affine transformation. (Middle) Deformation of the brain after MLG algorithm application. (Right) Field of deformation vectors. 


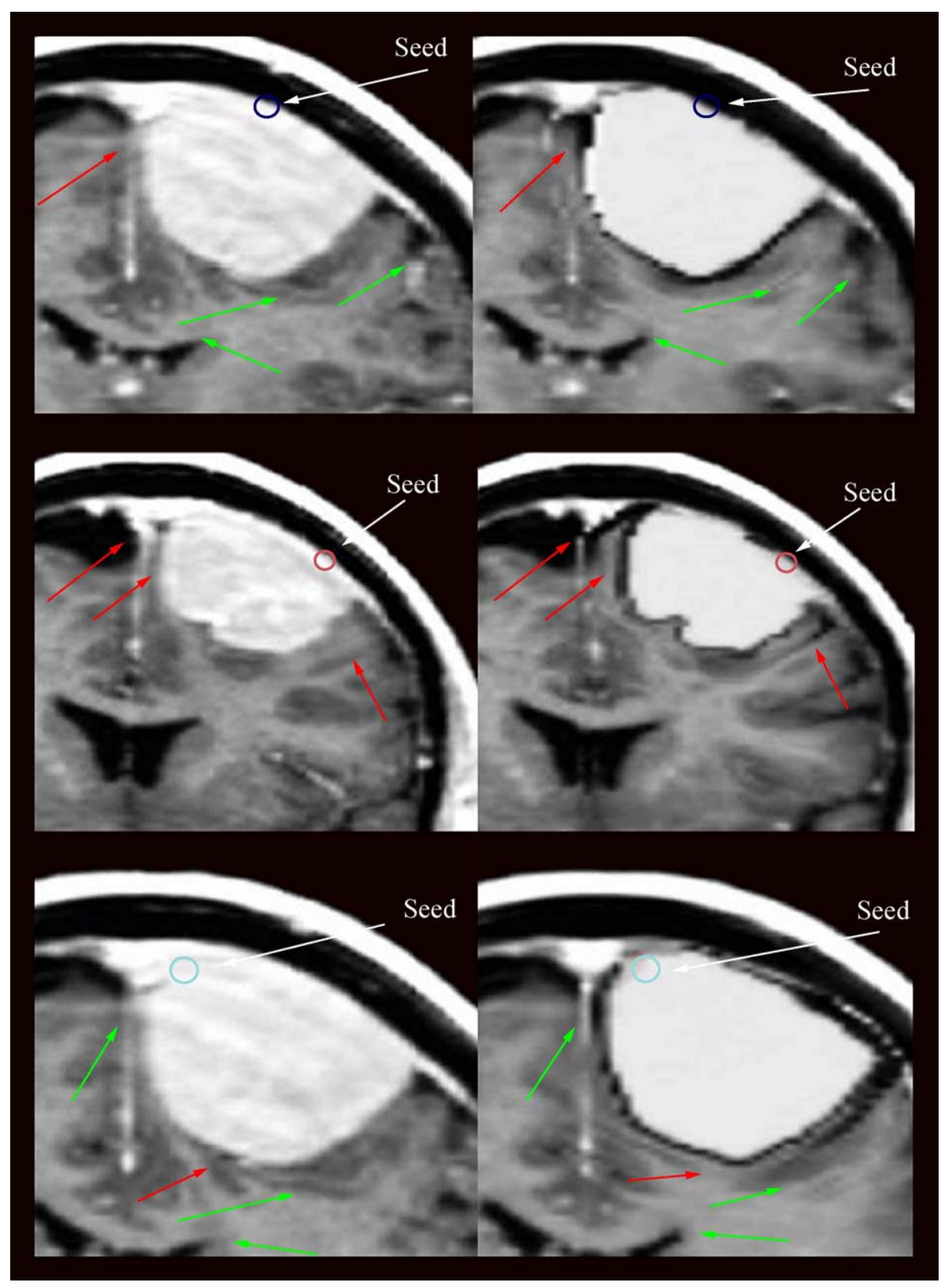

Fig. 4. Accuracy of deformation (right side) related to seeding point (circles) position compared to the patient MR images (left side).

erations. Several limitations have to be discussed in the highlight of potential clinical applications.

\section{Patient selection}

Both cases included in this study were meningiomas. Meningiomas typically exhibit a purely extracerebral growth with generally no associated cerebral edema and therefore induce a pure shift and deformation of the underlying brain structures, while intracerebral tumors may infiltrate the brain tissue and/or are often associated with brain edema. Our method does not yet apply for intracerebral space-occupying lesions that may require different models of tumor growth. Furthermore, due to their slow growth, meningiomas may reach significant volumes and induce significant displacements of the underlying brain structures before patients experience symptoms. The deformation algorithm accuracy could not yet be tested on such cases. Regarding therapeutic considerations, meningiomas of large volume are generally treated with surgery. However, radiosurgery or stereotactic radiotherapy may be indicated as a primary treatment or after an incomplete surgical removal in cases where tumor size does not exceed a maximal diameter of 25-30 $\mathrm{mm}$. Therefore, these case selection may be regarded as representative samples when radiosurgical treatment is considered.

\section{Hypothesis of radial growth}

The main hypothesis of the proposed method is that the lesion expands radially. Even if the vectors of growth of space-occupying lesions are not precisely known, it seems reasonable to assume from biological point of view that homogeneous intracerebral tumors (totally solid or totally cystic) have a radial growth. This is confirmed radiologically in cases where follow-up of such lesions 


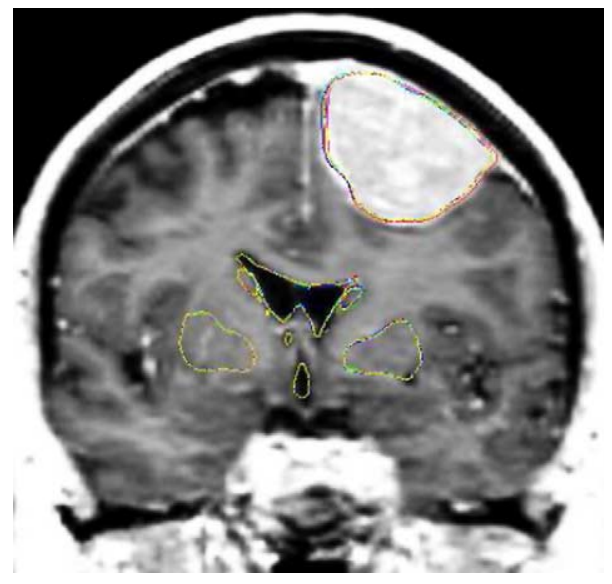

Fig. 5. Segmentation accuracy of basal ganglia and ventricles related to the seeding points shown in Fig. 4.

is performed. Regarding meningiomas, growth may be considered spatially homogeneous at least into directions where they are not restrained by anatomical structures like bone, cerebral falx, or tentorium. Actually, a large majority of meningiomas have a dural attachment, and dura, except the falx and tentorium, is adherent to bone. As bone is rigid, it is reasonable to consider that there is no growth into the bone direction, even if bone may be invaded in rare cases. It is then realistic to assume from a biological point of view that growth of meningiomas is radial and starts from the center of the surface of their dural attachment, defined as the seeding point.

\section{Importance of the seeding point position}

The deformation between atlas and patient compensates both for the tumor growth and for the interpatient variability, while the location of the seed is supposed to affect only the tumor growth model. Thus, the accuracy of brain deformation induced by the lesion growth is correlated with the position of the seeding point. This observation is not surprising considering the hypothesis of radial growth discussed earlier. As a result, the best deformation accuracy is not necessarily obtained when seeding point is, from a biological point of view, logically placed in the center of the surface of dural attachment. Indeed, no shift of either the falx or the bony sellar region is observed despite the presence of the meningioma, while the corresponding deformed images by the MLG method have deformed these structures. Actually, falx and tentorium, although less rigid than bone, have significantly higher resistances against tumor growth than brain and should be considered almost nondeformable structures until the tumor reaches a significant size. Consequently, in a future work, the algorithm should be modified in order to prevent too much deformability of these structures. Thus, a more adapted model of deformability could render the algorithm less dependent of finding the precise seed position.

\section{Control of the elasticity (adaptive Gaussian filter)}

In order to approximate the progressive decreasing deformation within the brain tissue as we move away from the tumor site, an adaptive Gaussian filter was applied to the deformation algorithm. As extensively explained previously, the values of sigma were fixed experimentally. $\sigma=0 \mathrm{~mm}$ inside the tumor area, where no regularization of the deformation field is needed, $\sigma=0.5 \mathrm{~mm}$ close to the tumor, allowing a maximal deformation in the surrounding brain structures, whereas $\sigma=0.8 \mathrm{~mm}$ away from the tumor in order to avoid large brain deformations distant from the tumor site. The choice of the $\sigma$ values was based on previous studies (Bach Cuadra, 2003), suggesting that a typical $\sigma$ for matching healthy brains ranges between 0.5 and $1 \mathrm{~mm}$. More

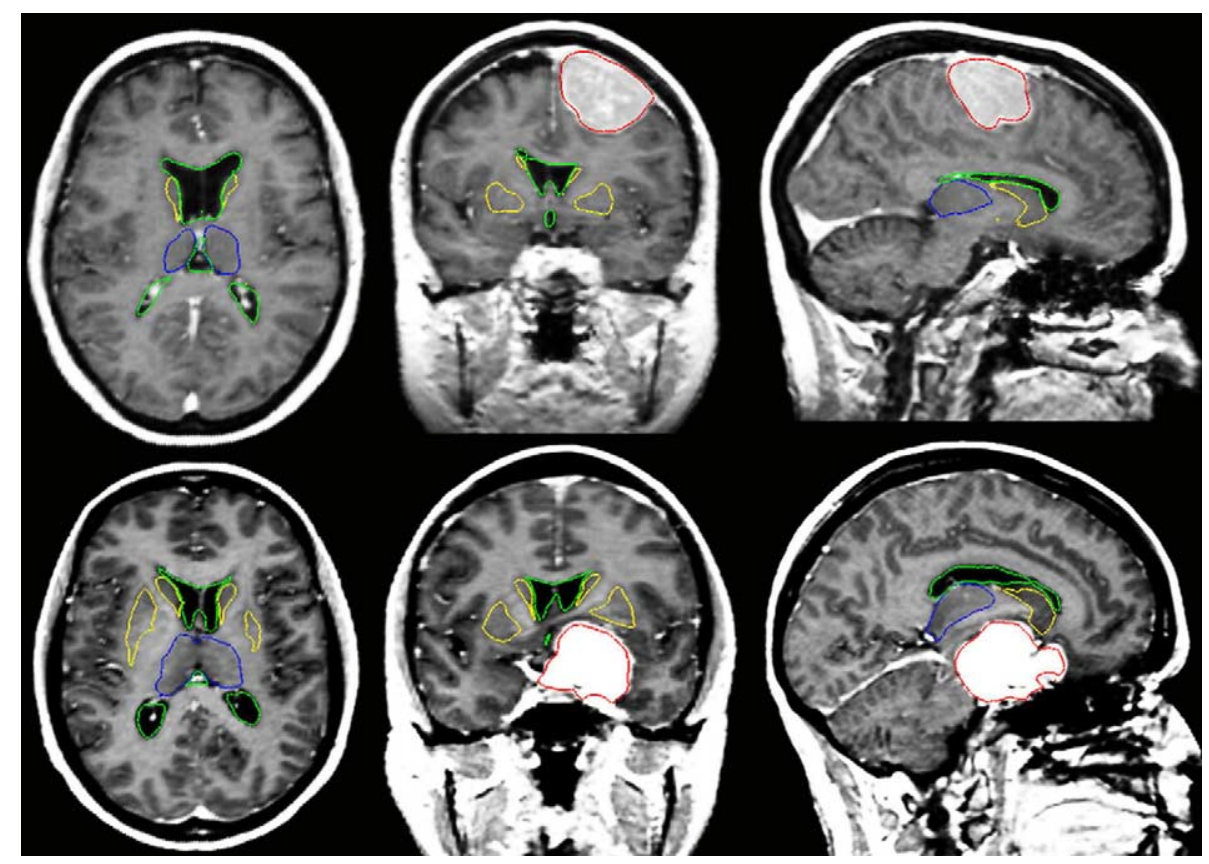

Fig. 6. Global segmentation results of the tumor (red), ventricles (green), thalamus (blue), and striatum (yellow) shown on an axial (left), coronal (center), and sagittal (right) image. Top: patient 1. Bottom: patient 2. 
accurate results may be obtained if sigma values could be adapted locally as proposed in other models of brain deformation (Ferrant et al., 2001).

\section{Control of the deformation accuracy}

Qualitative control of the deformation accuracy was relatively easy to perform for those segmented structures located away from the tumor site (ventricles, basal ganglia, thalamus), and segmentation accuracy of the basal ganglia and ventricles was consistent with patient visible MRI structure. However, it was more difficult to assess the accuracy of the deformation for structures surrounding the tumor, that is, the underlying brain gyri and sulci in cases of meningiomas. The displacement of reference points placed on the surrounding brain of the atlas or the contralateral hemisphere of the studied patients before deformation was studied, but it is almost impossible to give a precise interpretation on the position of these points after deformation when compared with the corresponding patient's images where these structures are already largely deformed. Moreover, interindividual (between patients) as well as intraindividual (between the two hemispheres of the same patient) variation of cortex anatomy may result in misinterpretations of the results.

\section{Conclusions}

We propose a new approach for brain deformation in presence of space-occupying tumors, based on a method for atlas-driven segmentation that uses a model of lesion growth that assumes, from a biological point of view, a radial expansion from a manual seeding point. It provides a minimal loss of information due to the minimal voxel size seeding and takes in consideration the progressive decreasing brain deformation as we move away from the tumor site. The preliminary results show that the segmented structures were consistent with the patient's anatomy when the deformation algorithm was implemented on extracerebral lesions inducing a pure displacement of the underlying brain structures. In this algorithm, deformation accuracy of surrounding brain structures was highly dependent on the accurate placement of the tumor seeding point. The validation and robustness of this method should be further assessed on a larger number of cases, including larger tumors as well as intracerebral tumors and lesions with cerebral edema.

Further improvements of the method should be obtained according to the biological and mechanical behavior of brain tumors and deforming brain structures that should be better understood by a multidisciplinary collaboration to improve the surgical, radiotherapeutical, and radiosurgical planning and treatment of space occupying brain lesions.

\section{References}

Bach Cuadra, M., 2003. Atlas-based segmentation and classification of magnetic resonance brain images. PhD thesis, Swiss Federal Institute of Technology, EPFL, CH-1015 Switzerland, November 2003. Thesis

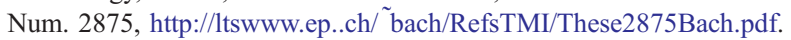

Bach Cuadra, M., Cuisenaire, O., Meuli, R., Thiran, J.-Ph., 2001. Automatic segmentation of internal structures of the brain in MRI using a tandem of affine and non-rigid registration of an anatomical atlas. International Conference in Image Processing (ICIP).

Bach Cuadra, M., Gomez, J., Hagmann, P., Pollo, C., Villemure, J.-G., Dawant, B.M., Thiran, J.-P., 2002. Atlas-based segmentation of pathological brains using a model of tumor growth. MICCAI, pp. $380-387$.

Cuisenaire, O., Thiran, J.-Ph., Macq, B., Michel, C., Volder, A.D., Marques, F., 1996. Automatic registration of 3D MR images with a computerized brain atlas. SPIE Med. Imaging 1719, 438-449.

Dawant, B.M., Hartmann, S.L., Thirion, J.-P., Maes, F., Vandermeulen, D., Demaerel, P., 1999a. Automatic 3-D segmentation of internal structures of the head in MR images using a combination of similarity and freeform transformations: I. Methodology and validation on normal subjects. IEEE Trans. Med. Imaging 18 (10), 909-916.

Dawant, B.M., Hartmann, S.L., Gadamsetty, S., 1999b. Brain atlas deformation in the presence of large space-occupying tumors. MICCAI, pp. $589-596$.

Ferrant, M., Nabavi, A., Macq, B., Jolesz, F.A., Kikinis, R., Warfield, S.K., 2001. Registration of 3-D intraoperative MR images of the brain using a finite-element biomechanical model. IEEE Trans. Med. Imaging 20 (12), 1384-1397.

Kaus, M.R., Warfield, S.K., Nabavi, A., Chatzidakis, E., Black, P.M., Jolesz, F.A., Kikinis, R., 1999. Segmentation of meningiomas and low grade gliomas in MRI. MICCAI, pp. 1-10.

Kikinis, R., Shenton, M.E., Iosifescu, D.V., McCarley, R.W., Saiviroonporn, P., Hokama, H.H., Robatino, A., Metcalf, D., Wible, C.G., Portas, C.M., Donnino, R., Jolesz, F.A., 1996. A digital brain atlas for surgical planning, model driven segmentation and teaching. IEEE Trans. Vis. Comput. Graph. 2 (3), 232-241.

Thirion, J.-P., 1995. Fast non-rigid matching of 3D medical images. Tech. Rep. 2547, INRIA.

Thirion, J.P., 1998. Image matching as a diffusion process: an analogy with Maxwell's demons. Med. Image Anal. 2, 243-260.

Warfield, S.K., Kaus, M., Jolesz, F.A., Kikinis, R., 2000. Adaptive, template moderated, spatially varying statistical classification. Med. Image Anal. 4, 43-55. 